\title{
Sistema Heráclito: Objeto de Aprendizagem para o Ensino da Dedução Natural na Lógica Proposicional
}

\author{
${ }^{1}$ Fabiane Flores Penteado Galafassi, ${ }^{1}$ João Carlos Gluz, ${ }^{1}$ Lucas Gomes, Marcel \\ ${ }^{1}$ Mossmann \\ ${ }^{1}$ Programa Interdisciplinar de Pós Graduação Em Computação Aplicada (PIPCA) - \\ Universidade do Vale do Rio dos Sinos (UNISINOS) - Caixa Postal 275 - 93.022-000 \\ - São Leopoldo - RS - Brasil \\ \{fabiane.penteado, marcelmossmann\}@gmail.com, jcgluz@unisinos.br, \\ lucas.gomes.rs@hotmail.com
}

\begin{abstract}
This article aims to present the system of Heraclitus and his publisher evidence. Characterized as a learning object, the system addresses the teaching of Heraclitus natural deduction in propositional logic. Focuses associated with the use of agent technologies with pedagogical features that make the use of different teaching and learning strategies in order to assist the student in his reasoning process in solving an exercise. Preliminary experiments in the classroom showed that the tool got good acceptance and tutoring system is efficient.
\end{abstract}

Resumo. O presente artigo tem como objetivo apresentar o sistema Heráclito e seu editor de provas. Caracterizado como um objeto de aprendizagem, o sistema Heráclito aborda o ensino da dedução natural na lógica proposicional. Tem seu foco associado ao uso de tecnologias de agentes com características pedagógicas que fazem o uso de diversas estratégias de ensino-aprendizagem com o intuito de auxiliar o aluno em seu processo de raciocínio na resolução de um exercício. Experimentos preliminares em sala de aula mostraram que a ferramenta obteve boa aceitação e o sistema tutor se mostrou eficiente.

\section{Introdução}

O problema científico mais geral que o Sistema Heráclito aborda é um problema de Ensino Superior, vinculado aos cursos de Bacharelado e Licenciatura em Computação, que incluem, entre outros, cursos como Ciência da Computação, Engenharia da Computação e Sistemas de Informação. Com a definição do currículo base de ensino destes cursos proposta pela SBC (Sociedade Brasileira de Computação) que foi adotada pelas diretrizes curriculares do MEC (Ministério da Educação e Cultura), a disciplina de Lógica (também denominada de Lógica para Computação, Lógica Matemática ou Lógica Formal) passou a ser considerada matéria (conteúdo) básica dos referidos cursos, tipicamente compondo a grade curricular do primeiro ou segundo semestre dos cursos.

A disciplina de Lógica é fundamental para a formação dos alunos de Computação e Informática, ajudando-os no desenvolvimento de suas habilidades de análise lógica, formalização e resolução de problemas. Estas habilidades, por sua vez, são necessárias para as atividades tão diversas quanto programação, análise e especificação de requisitos, projeto de bancos de dados, dentre outras áreas da Computação e da Informática.

Nesse sentido o Sistema Heráclito se apresenta aos seus usuários como um 
objeto de aprendizagem que têm por objetivo auxiliar os alunos da disciplina de Lógica e seu editor tem como principal funcionalidade auxiliar na elaboração de provas de argumentos formais por meio das regras da Dedução Natural. Atualmente se apresenta em duas versões: Desktop/Laptop e Móvel (Tablets e Smartphones).

\section{Objetos de Aprendizagem Heráclito}

Um objeto de aprendizagem Heráclito na versão Desktop/Laptop consiste em um applet Java e pode ser obtido diretamente de um ambiente virtual de aprendizagem (caso o professor esteja utilizando-o como ferramenta de apoio na disciplina) ou através do portal OBAAMILOS ${ }^{1}$. Esse applet contém: o aplicativo Editor de Provas e seu manual de utilização, listas de exercícios preparadas por um professor, exemplos de demonstrações corretas e materiais (tanto em forma gráfica quanto textual) explicativos sobre as listas de exercícios e conteúdos de Lógica tratados pelo objeto. Já um objeto de aprendizagem Heráclito na versão Móvel (Tablets e Smartphones) consiste em um aplicativo Android e também pode ser obtido diretamente de um ambiente virtual de aprendizagem (caso o professor esteja utilizando-o como ferramenta de apoio na disciplina) ou através do portal OBAAMILOS. Esse aplicativo contém exatamente o mesmo pacote de conteúdo encontrado na versão Desktop [Galafassi, 2012].

\section{Arquitetura do Sistema Heráclito}

A arquitetura de software definida para o Sistema Heráclito, Figura 1, é compatível com a infraestrutura de agentes MILOS do projeto OBAAMILOS [Vicari et. al., 2009] e possui três agentes: Perfil do Aluno, Mediado e Especialista.

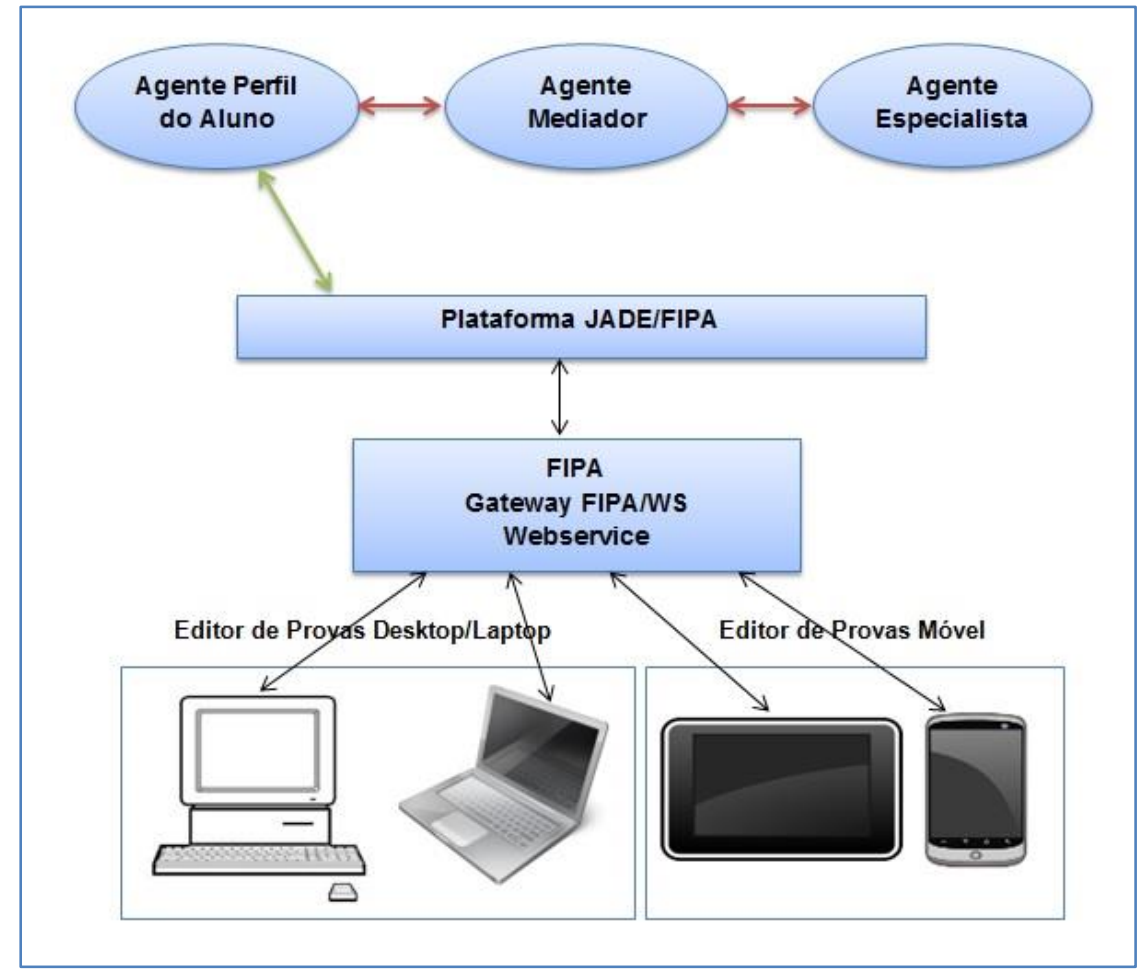

Figura 1. Arquitetura do Sistema Heráclito.

Fonte: Elaborado pela autora e demais integrantes do projeto OBAAMILOS.

\footnotetext{
${ }^{1}$ O Portal MILOS está disponível em: http://obaa.unisinos.br/.
} 
- Agente Perfil do Aluno: Este agente representa o modelo de aluno ${ }^{2}$, que no Sistema Heráclito é baseado no processo de resolução de exercícios de dedução. Conforme [Dillenbourg e Self, 1992] um modelo de aluno pode ser analisado em três níveis de abstração: operacional, comportamental e conceitual. Aplicando-se essa análise ao ensino de dedução na Lógica obtêm-se modelos com a seguinte organização:

(1) Nível Operacional: neste nível o modelo é responsável por verificar se o aluno consegue ou não aplicar as regras de dedução de forma correta.

(2) Nível Comportamental: o modelo avalia se o aluno consegue ser proficiente em seu processo de raciocínio para resolver um dado problema de dedução na Lógica.

(3) Nível Conceitual: o modelo indica a assimilação dos conceitos envolvidos no processo de dedução na Lógica. É atingido quando o aluno compreende as implicações da dedução para outras áreas da Lógica e para áreas relacionadas como a Matemática e a Ciência da Computação.

O agente Perfil do Aluno do Heráclito trabalha nos níveis operacional e comportamental, verificando se o aluno sabe aplicar corretamente as regras de dedução e se ele é capaz de avançar no processo de resolução de um exercício de dedução até o final. Nesta última tarefa o agente Perfil do Aluno conta com a ajuda do agente Especialista do Heráclito.

O agente Perfil do Aluno ainda possui outras tarefas adicionais, tais como: guardar o histórico por aluno, gerando um registro de ações $(\log )$ para que o professor possa consultar posteriormente, e para que o agente Mediador também possa consultar, para fins de decisão em seu processo pedagógico de mediação. Em outras palavras, este agente deve manter as informações relacionadas ao aluno em questão.

- Agente Mediador: Este agente representa o papel do professor em sua função didático-pedagógica, fazendo uso de diversas estratégias de ensinoaprendizagem com o intuito de auxiliar o aluno em seu processo de raciocínio na resolução de um exercício.

- Agente Especialista: Este agente também representa o papel do professor, mas em sua função de especialista no domínio de ensino de Lógica. Este agente segue o processo de resolução do aluno acompanhando a prova que o aluno está resolvendo. Este agente tem a capacidade de resolver o problema em questão do ponto em que o aluno está trabalhando até seu final. Com essa capacidade o agente Especialista pode detectar se a aplicação dos passos de dedução natural segue corretamente ou não. Este agente possui uma interface em Java e trabalha em conjunto com o provador de teoremas. Este provador foi desenvolvido em Prolog e é responsável por realizar as provas dos argumentos inseridas no sistema Heráclito.

Todas as mensagens trocadas entre esses três agentes passam obrigatoriamente pelo

\footnotetext{
${ }^{2} \mathrm{O}$ perfil deste agente representa um modelo de aluno que no sistema Heráclito foi definido com base no processo de resolução de exercícios de dedução, ou seja, ele representa o comportamento do aluno dentro do processo de resolução dos exercícios, juntamente com as possíveis ações que o aluno poderá realizar, visto que estas ações podem ser previamente previstas.
} 
agente mediador, que é quem media a comunicação e toma as decisões [Galafassi, 2012].

\section{Editor de provas do Sistema Heráclito - Versão Desktop/Laptop}

O Heráclito versão Desktop/Laptop tem em sua tela inicial de boas vindas, quatro botões com opções distintas e um espaço para o usuário realizar o Login de usuário como pode ser visto na Figura 2:

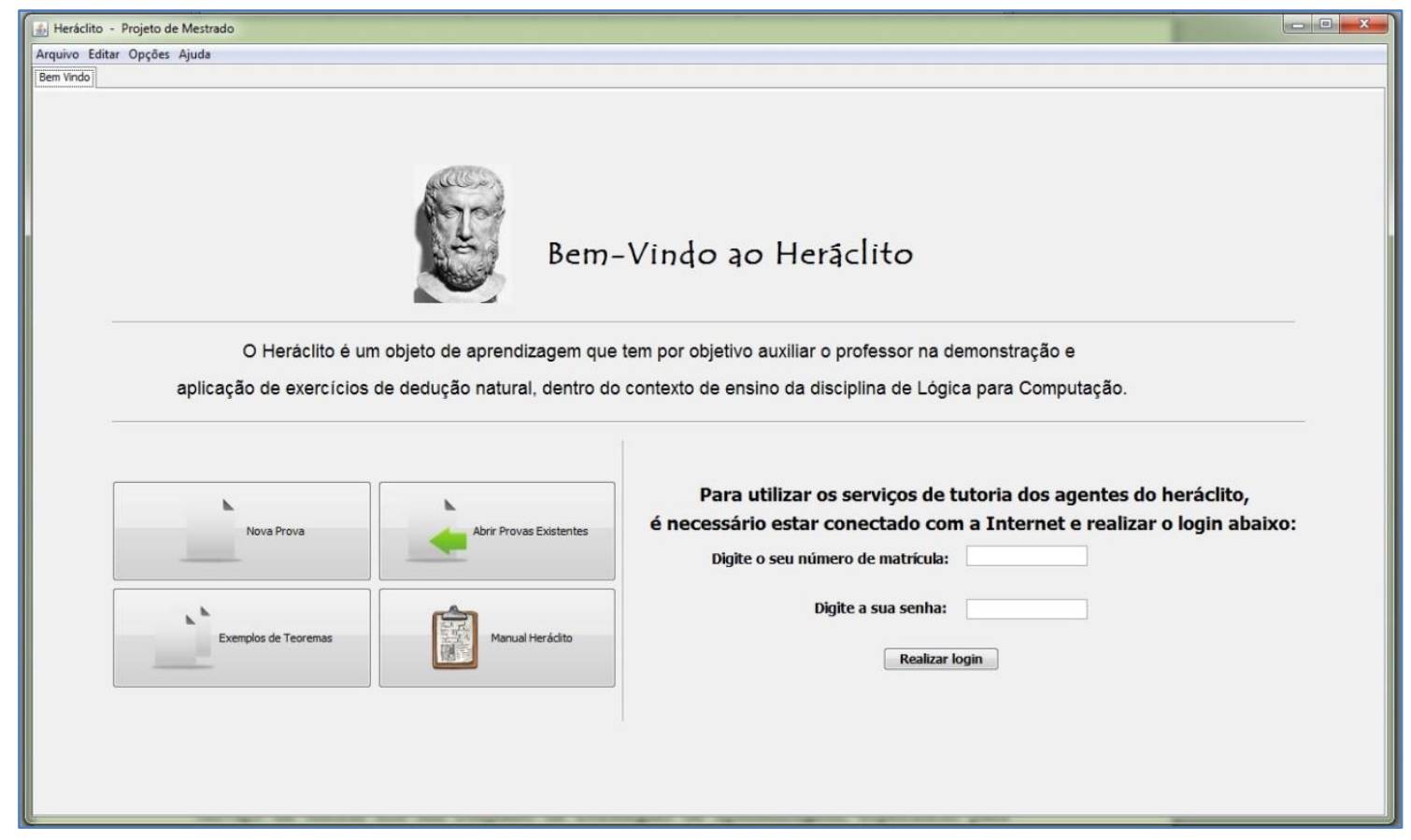

Figura 2. Tela inicial do Sistema Heráclito.

Cada botão exerce uma funcionalidade, sendo eles:

- Botão Nova Prova: Esta opção permite ao usuário iniciar uma nova prova a partir de um conjunto de opções de exercícios de demonstração selecionados pelo professor. É onde as edições das provas de lógica são realizadas. Uma vez instalado no computador do aluno, o Heráclito se comportará como um editor de provas que permite ao aluno resolver os problemas de demonstração.

- Botão Exemplos de Provas: Esta opção dá acesso a argumentos já provados, elaborados pelo professor e é composta por uma série de exercícios que foram divididos em 3 níveis de complexidade: inicial, intermediaria e avançada.

- Botão Abrir Provas Existentes: Esta opção dá acesso às provas feitas pelo aluno que foram testadas e salvas no computador (podendo ser abertas ou retomadas a qualquer momento).

- Botão Manual do Heráclito: manual de instruções e de funcionamento da ferramenta.

O editor pode operar em dois modos: o modo local onde não é oferecido serviço de tutoria, ou seja, não é necessário realizar login e o modo conectado, onde o usuário 
realiza login (com usuário e senha previamente cadastrados ${ }^{3}$ ) e tem disponível o suporte pedagógico por meio dos agentes de apoio pedagógico para o ensino de Lógica, disponibilizados pelo servidor MILOS.

Ao realizar login no sistema Heráclito, o usuário estará conectado com o servidor, podendo usufruir dos serviços oferecidos pela ferramenta. Após a confirmação da conexão já é possível usar o sistema escolhendo um dos quatro botões. O serviço de tutoria usa um conjunto de estratégias de aprendizagem, específicas para Lógica, necessárias para auxiliar o aluno na realização de uma prova de Dedução Natural.

A principal interface da ferramenta Heráclito é a tela de edição de provas e argumentos (acionada por meio da interface inicial do sistema no botão nova prova). Uma vez selecionado o botão Nova Prova, uma segunda tela com edição de provas será aberta (em abas). É nesta tela que os argumentos formais são provados. Esta tela ainda é composta por botões laterais (do lado esquerdo) os quais representam as regras de inferências básicas e derivadas, como pode ser visto na Figura 3 [Galafassi, 2012].

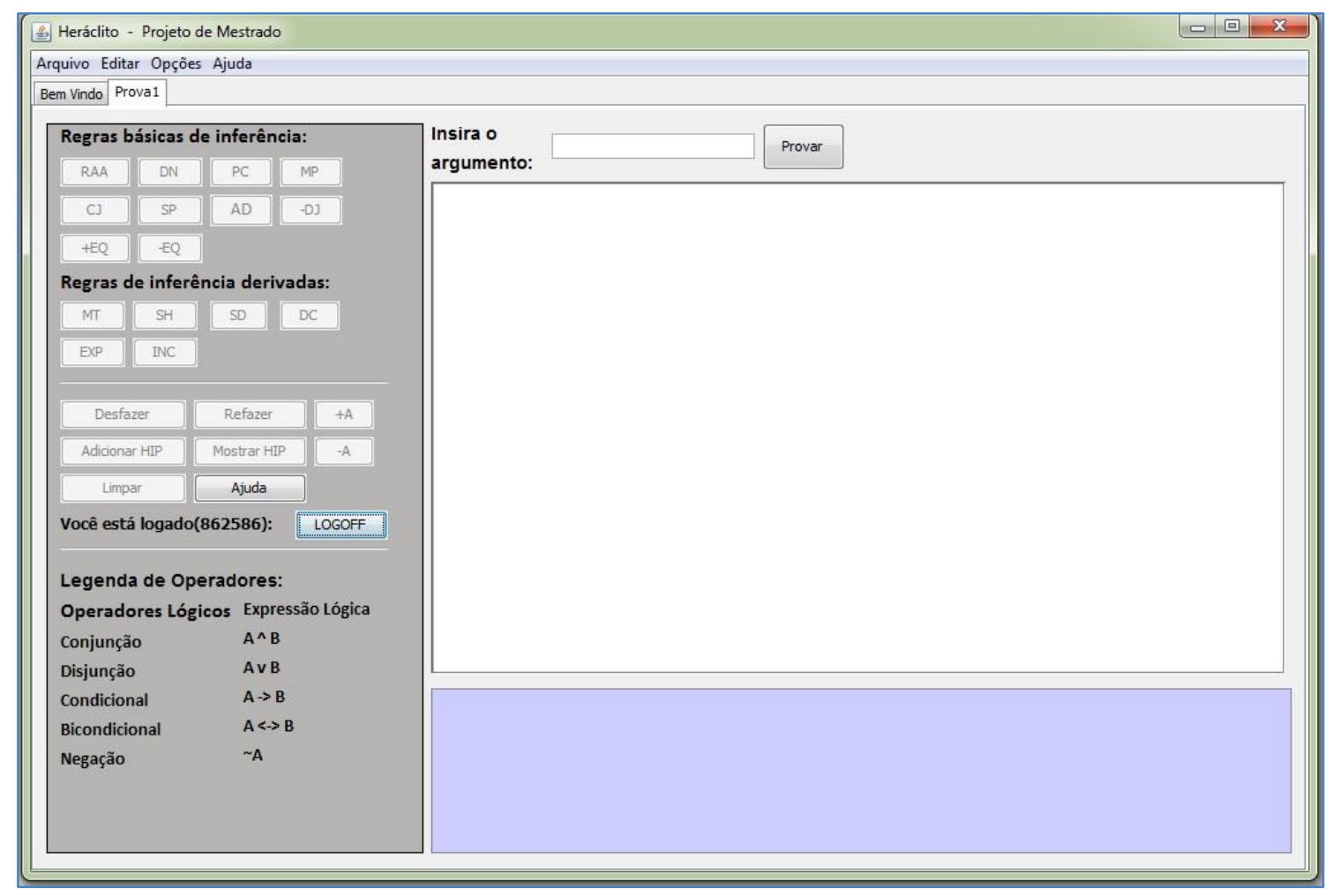

Figura 3. Tela de edição do Sistema Heráclito

A demonstração é elaborada passo-a-passo com base na aplicação das regras de inferência (básica e derivada). São oito regras básicas de inferência não hipotética:

- MP (modus ponens);

- DN (dupla negação ou eliminação Da negação);

- CJ (introdução da conjunção);

- SP (separação ou eliminação da conjunção);

- $\mathrm{AD}$ (adição ou introdução da disjunção);

- DJ (eliminação da disjunção);

\footnotetext{
${ }^{3}$ Para usar os serviços de tutoria do Heráclito é necessário realizar login. Em sua atual fase, o usuário é cadastrado previamente e recebe um usuário e uma senha do sistema.
} 
- $\quad$ EQ (introdução da equivalência ou do bicondicional);

- -EQ (eliminação da equivalência ou do bicondicional).

E duas regras hipotéticas:

- RAA (redução ao absurdo) e PC (prova condicional), além das regras derivadas:

- MT (modus tolens);

- SH (silogismo hipotético);

- SD (silogismo disjuntivo);

- DC (dilema construtivo);

- INC (inconsistência) e

- EXP (exportação).

\section{Editor de provas do Sistema Heráclito - Versão Móvel (Tablets e Smartphones)}

O Heráclito versão móvel apresenta em sua tela inicial o diálogo de login, onde um usuário e uma senha serão solicitados. Caso o aluno não possua um login o botão cancelar deverá ser pressionado. A versão móvel também pode operar em dois modos: o modo local onde não é oferecido serviço de tutoria, ou seja, não é necessário realizar login e o modo conectado, onde o usuário realiza login (com usuário e senha previamente cadastrados) e tem disponível o suporte pedagógico por meio dos agentes de apoio pedagógico para o ensino de Lógica, disponibilizados pelo servidor MILOS.

Assim como na versão Desktop, ao realizar login no sistema Heráclito, o usuário já estará conectado com o servidor, podendo usufruir dos serviços oferecidos pela ferramenta e o serviço de tutoria.

A principal interface da ferramenta Heráclito versão móvel é a tela de edição de provas e argumentos e pode ser vista na Figura 4 [Galafassi, 2012].

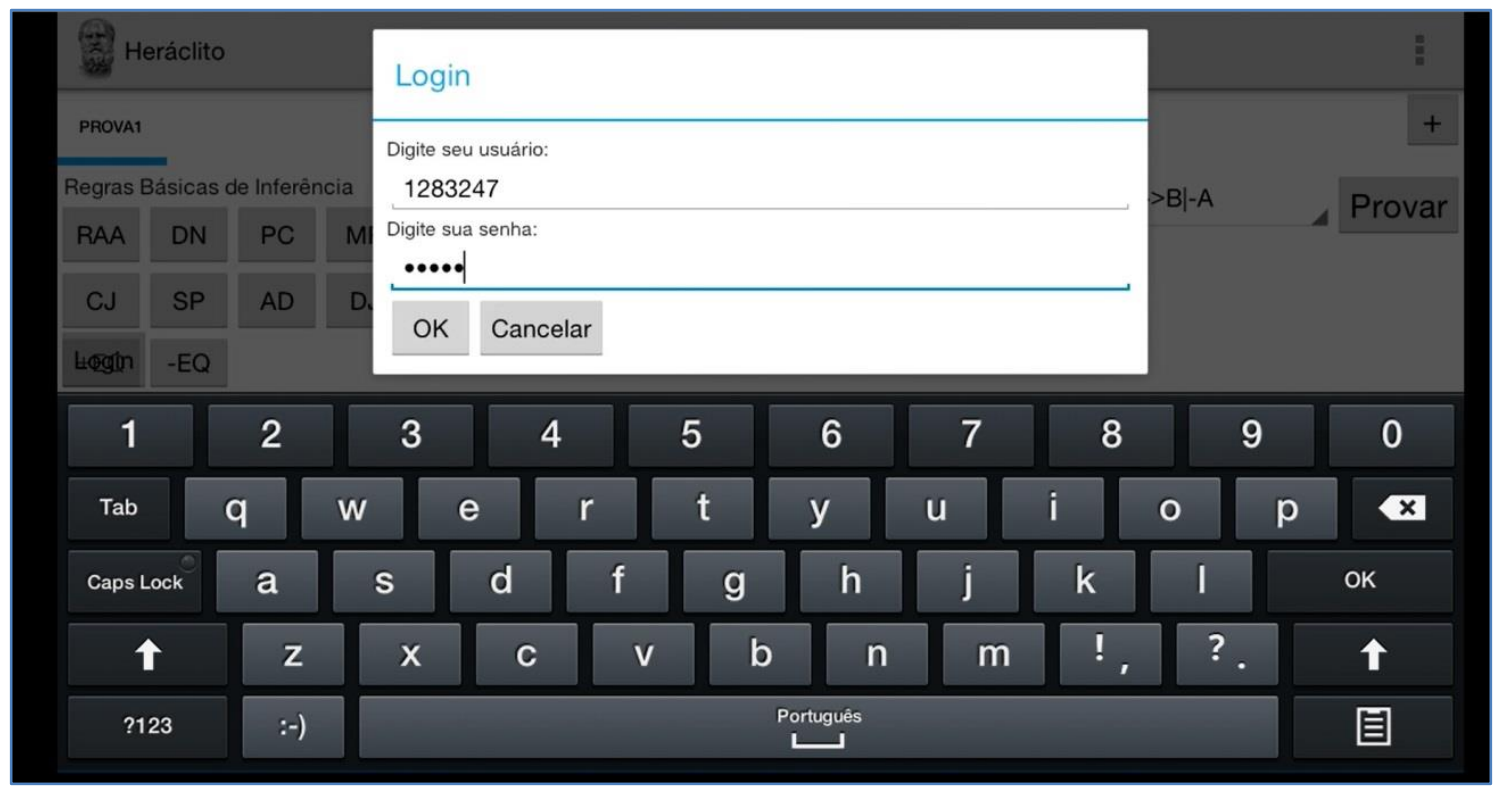

Figura 4. Interface da versão móvel do sistema Heráclito

A versão móvel se diferencia da versão Desktop no sentido que uma vez conectado, já é possível utilizar o sistema e, na versão Desktop é necessário selecionar o botão "Nova Prova". Para abrir uma nova prova/argumento basta pressionar o botão 
com símbolo + e uma segunda tela com edição de provas será aberta (em abas). São nestas telas (abas) que os argumentos formais são provados. Esta tela ainda é composta por botões laterais (do lado esquerdo) os quais representam as regras de inferências básicas e derivadas. A demonstração é elaborada passo-a-passo com base na aplicação das regras de inferência (básica e derivada) e possuem o mesmo conjunto de regras encontrado na versão Desktop.

\subsection{A Tutoria no Heráclito}

O serviço de tutoria do Heráclito visa ajudar o aluno na resolução dos exercícios, fazendo o papel do professor. À medida que o aluno for usando o Heráclito na resolução dos exercícios, os agentes pedagógicos do serviço de tutoria estarão monitorando suas ações, prontos para auxiliá-los em caso de necessidade. Se o aluno não conseguir avançar na realização de uma prova, este poderá solicitar ajuda ao tutor por meio do botão Ajuda. O botão Ajuda também poderá ser acionado a qualquer momento da prova. O serviço de tutoria usa um conjunto de estratégias de aprendizagem, específicas para Lógica, ao ajudar o aluno a realizar uma prova de Dedução Natural [Galafassi, 2012].

\subsection{Editor de Provas em Modo Local}

O modo local do editor de provas não possui acesso ao serviço de tutoria e este é usado quando o aluno não realiza o processo de login. Entretanto, o editor de Provas ainda é capaz de tratar algumas situações que ocorrem durante a resolução de um problema de dedução. São elas [Galafassi, 2012]:

- Verificação da inserção de hipóteses: o editor é responsável pela verificação se a inserção é correta ou não. Ao inserir uma hipótese, o Editor verifica se a hipótese é correta ou não. Uma hipótese é correta quando faz parte da lista de hipóteses do argumento.

- Verificação de aplicação de regras de dedução: o editor simplesmente não permite que o aluno insira um novo passo através da aplicação incorreta de uma regra de dedução.

- Em modo local o botão Ajuda provê acesso ao manual do editor e a exemplos de provas previamente resolvidas. O exemplo é escolhido aleatoriamente porque o Editor não possui suporte do serviço de tutoria para saber qual exemplo de uso de regra de dedução sugerir para o próximo passo.

\section{Agente Mediador}

O agente Mediador é responsável por incorporar o papel do professor ou tutor dentro do Sistema Heráclito, provendo um apoio pedagógico ao aluno em seu processo de aprendizagem da dedução natural na Lógica Proposicional. Este apoio pode ser dado em várias situações deste processo.

Para todas essas situações o agente Mediador fica responsável pela efetiva intervenção de auxílio neste processo. Além destas situações, caso o aluno cometa um erro ou venha repetir o mesmo erro, o agente Mediador seleciona estratégias pedagógicas apropriadas para tratar do erro. 
A seguir são apresentadas as principais situações em que o agente Mediador irá interferir através da aplicação de alguma estratégia de mediação [Galafassi, 2012]:

- Quando ocorre uma inserção incorreta de hipóteses;

- Quando ocorre uma aplicação incorreta de regra de dedução;

- Quando ocorre uma aplicação correta (no nível operacional) de uma regra: neste caso, mesmo a aplicação sendo correta no nível operacional esta regra ainda pode ser problemática no nível comportamental, podendo ser categorizada em útil, redundante e prejudicial. A aplicação de uma regra útil supõe que a regra está correta (operacionalmente) e contribui para a resolução do exercício. A aplicação de uma regra redundante pode estar correta no nível operacional, mas não contribui com a resolução do exercício, e o aluno apenas aumenta o número de linhas e regras aplicadas sem obter o resultado esperado. E a regra prejudicial quando aplicada acaba por levar o aluno a um caminho sem volta, ou seja, com a sua aplicação o exercício não poderá ser resolvido;

- Quando o aluno fica muito tempo ocioso ou sem avançar na resolução do exercício;

- Quando se atinge um percentual significativo da resolução da prova o aluno é informado e recebe um incentivo para prosseguir;

- Quando faltam poucos passos (tipicamente 1 ou 2 passos) para a resolução da prova o aluno é informado e recebe um incentivo para finalizar;

- Quando o aluno solicita ajuda. As respostas para as solicitações de ajuda podem ser de dois tipos: um exemplo de demonstração apropriado para a situação atual do aluno, ou, caso a situação do aluno seja mais crítica, uma dica, onde o sistema fornece o próximo passo a ser feito para continuar no processo de resolução.

\section{Testes/Experimentos e trabalhos futuros}

Para avaliar experimentalmente o sistema, foi apresentado o objeto de aprendizagem Heráclito contendo o Editor de Provas e seu guia de utilização, uma lista com 10 de exercícios de dedução natural (divididos em três níveis de dificuldade em relação ao seu processo de resolução) e um conjunto de exemplos de dedução, relacionado aos exercícios a serem resolvidos pelos alunos.

Foi solicitado aos alunos que estes executassem o experimento em duas etapas: primeiro resolver os exercícios da lista de exercícios com o uso do objeto de aprendizagem Heráclito e posteriormente preencher um questionário de avaliação sobre a utilização do mesmo, onde se buscou avaliar a percepção dos alunos a respeito do uso do Heráclito. Os principais critérios foram: efetividade pedagógica da Mediação, a qualidade do conteúdo, o layout visual e a usabilidade da ferramenta.

Realizado em uma instituição de ensino superior com uma turma de graduação da disciplina de Lógica no semestre de 2012/2. Os testes tiveram inicio após terem sido cobertos os conteúdos de operadores e fórmulas, tabelas-verdade e processo de dedução natural na Lógica Proposicional, logo após o a primeira avaliação formal. Os alunos já haviam trabalhado em listas de exercício similares, mas com resolução manual e acompanhamento do professor.

O experimento foi iniciado em um laboratório de informática com a apresentação do objeto de aprendizagem de teste Heráclito e a orientação dos alunos sobre a ferramenta, tais como, recursos, funcionalidades e formas de acesso. Os testes tiveram início no laboratório e posteriormente foram terminados pelos alunos em casa. 
Foi dado um período de 10 dias para a condução do experimento. O processo de uso e resolução dos exercícios foi registrado por meio dos registros de ações $(\log s)$ do sistema.

De uma turma com 35 alunos participantes do experimento, 22 efetivamente utilizaram o Sistema Heráclito e destes 22 alunos, 13 foram aprovados e 9 foram reprovados. Cada aluno decidiu por conta própria se queria ou não participar do experimento.

Com relação à resolução dos exercícios pelos alunos, obteve-se um total de 135 exercícios realizados (dados extraídos dos logs) com a ajuda do Sistema Heráclito. Destes 135, 69 deles eram considerados de nível baixo, 43 deles eram de nível médio e 23 considerados de nível difícil.

Deste experimento pode-se concluir que as estratégias de ensino desenvolvidas neste trabalho se mostraram capazes de fornecer uma importante ajuda no processo de aprendizado da disciplina de Lógica, como aponta a média de $69 \%$ de aprovação sem nenhum tipo de restrição. O somatório com aprovações parciais que resultou em $92 \%$ de aprovação, demonstrando claramente que a mediação exerce um papel fundamental no processo de ensino-aprendizagem. É importante notar que esse índice resulta da percepção dos alunos que efetivamente interagiram com o sistema. Isso fornece indícios sólidos que o Sistema Heráclito consegue transparecer aos alunos que é realmente um tutor capaz de ajudar na aprendizagem de dedução na Lógica Proposicional.

Essa é uma tarefa importante (e difícil) nos processos de ensino-aprendizagem, pois qualquer professor ou tutor humano sabe como é importante ganhar a confiança dos alunos, mostrando que é capaz de ajudá-los a aprender determinado tópico de ensino. Os índices de aprovação vistos acima mostram que o processo de mediação (tutoria) do Sistema Heráclito está, ao menos, no caminho certo (a versão completa e detalhada deste experimento pode ser vista em [Galafassi, 2012]).

No que diz respeito à utilização da ferramenta, os alunos se mostraram bastantes favoráveis como demonstrado nas questões onde se pergunta "Como aluno você acredita que o Heráclito de uma forma geral tenha facilitado o aprendizado?" e "Os serviços de tutoria oferecida pelo Heráclito contribuíram para uma melhor compreensão do desenvolvimento dos exercícios?". As respostas a essas perguntas mostram que os alunos acreditam que o sistema tutor facilitou o processo de aprendizado e que também contribuiu para uma melhor compreensão do desenvolvimento dos exercícios.

Essas avaliações dos alunos fornecem indícios importantes que a utilização do sistema Heráclito poderá contribuir para a melhora dos níveis de aprovação. A verificação se essa melhora se confirma ou não é um importante trabalho futuro de pesquisa sobre o Sistema Heráclito.

Por fim, o Layout e Usabilidade se mostraram favoráveis à adesão da ferramenta por parte dos alunos, uma vez que eles não tiveram maiores problemas em entender o sistema Tutor e a interação realizada por meio de suas mensagens.

Como sugestão para melhoramento da ferramenta tem-se algumas funcionalidades sugeridas por parte dos alunos tais como: a opção de mostrar em tela a correta utilização da regra. Isso poderia ser feito por meio do posicionamento do mouse em cima da regra, de modo que o sistema mostraria a utilização da regra, eliminando a necessidade de acessar o manual (ou outro meio) para identificar o uso de determinada regra. Outras sugestões de melhorias acolhidas foram com relação à gravação dos exercícios e aos tipos de regras a serem utilizadas. Quanto aos exercícios realizados, busca-se uma forma onde os dados estejam disponíveis para o usuário acessá-los e analisa-los posteriormente (até para o aluno saber quais exercícios já realizou). Quanto aos tipos de regras a serem aplicadas, os alunos acreditam que o processo de 
aprendizagem pode ser melhorado se eles forem informados quando uma regra básica de dedução ou uma regra derivada deve ser aplicada, no lugar da sugestão explícita da regra. Acredita-se que com o sistema auxiliando neste ponto os alunos passariam a desenvolver melhor a sua percepção e visão quanto ao uso das regras.

As últimas sugestões estão relacionadas à usabilidade, sendo estas de ordem mais técnica, como a compatibilidade entre sistemas operacionais, o auto ajuste do sistema em resoluções distintas, bem como as funcionalidades citadas acima que já estão em fase de desenvolvimento.

Quanto a trabalhos futuros, conforme comentado antes, mostra-se importante fazer uma avaliação para confirmar a expectativa acerca de melhoras no processo de ensino-aprendizagem fazendo uma análise experimental sobre a eficácia do apoio ao ensino, preferencialmente com a utilização do grupo (ou possivelmente turma) de controle. Note-se que, como qualquer processo de avaliação de um método de ensino, tais experimentos para a medida de eficácia são sempre muito subjetivos e relativos ao contexto em que são aplicados, não fornecendo comprovações ou certificações sobre a qualidade do método em geral. O processo de mediação no Sistema Heráclito é um método de ensino implementado computacionalmente, sofrendo das mesmas dificuldades de avaliação de sua eficácia. Apesar disso, tais experimentos podem contribuir para uma melhor compreensão sobre as capacidades do processo de mediação e sobre como ele pode ser usado de forma mais proficiente em contextos de ensino.

\section{Referencias}

Dillenbourg, P., \& SELF, J. (1992) People Power: A human-computer collaborative learning system. In Intelligent Tutoring Systems (651-660).

Galafassi, Fabiane F. P. (2012) Agente Pedagógico para Mediação do Processo de Ensino-Aprendizagem da Dedução Natural na Lógica Proposicional (Dissertação de Mestrado). Programa Interdisciplinar de Pós Graduação Em Computação Aplicada (PIPCA) - Universidade do Vale do Rio dos Sinos (UNISINOS).

Vicari, R.; Gluz, J.; Santos, E.; et al. (2009) Projeto OBAA - Rel. Técnico RTOBAA01 - Proposta de Padrão para Metadados de Objetos de Aprendizagem Multiplataforma. UFRGS/CINTED. Disponível em: $<$ http://www.portalobaa.org/obaac/padraoobaa/relatoriostecnicos/RTOBAA01.pdf/view $>$. 